\title{
Dario Grossi (June 6, 1950-June 20, 2021)
}

\author{
Luigi Trojano $^{1} \cdot$ Elena Salvatore ${ }^{2}$
}

Accepted: 9 July 2021 / Published online: 2 August 2021

(c) Fondazione Società Italiana di Neurologia 2021

Born in Naples on June 6, 1950, Professor Dario Grossi passed away in Naples on June 20, 2021.

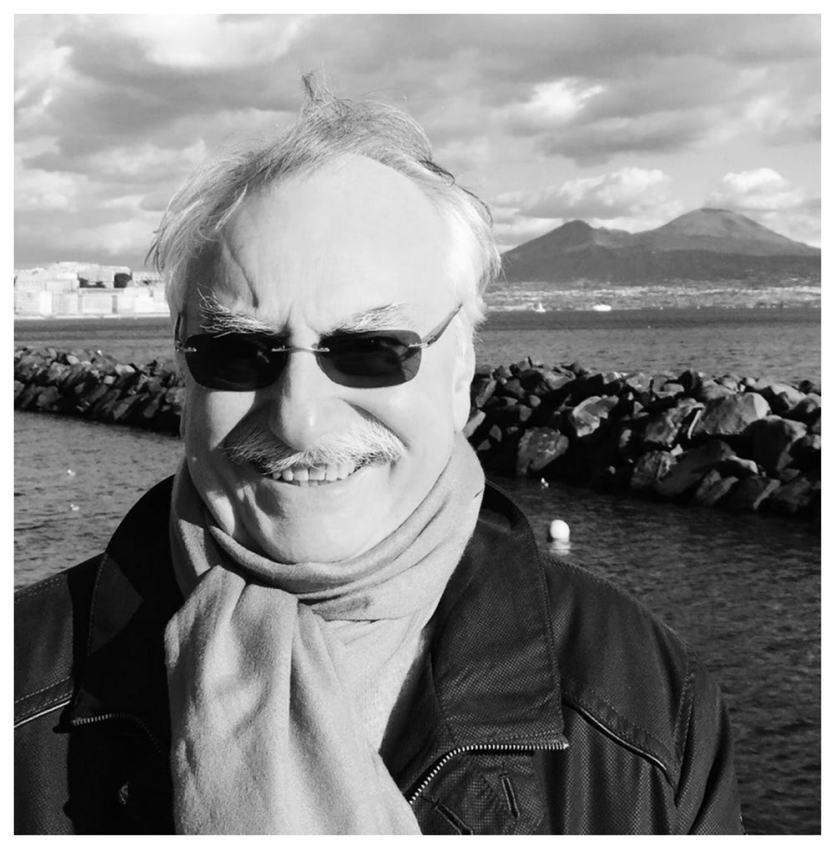

Dario Grossi, in Naples, January 2016. Photo provided by family

Professor Dario Grossi achieved his MD degree in 1974 at the University of Naples, where he also got his specialization in neurology (in 1978) and in psychiatry (in 1982). He started his academic career as a young researcher at the Clinica Neurologica of the then-called 'Second School of Medicine' of the University of Naples Federico II, directed

Luigi Trojano

luigi.trojano@unicampania.it

Elena Salvatore

elena.salvatore@unina.it

1 University of Campania "Luigi Vanvitelli”, Caserta, Italy

2 University of Naples "Federico II", Naples, Italy by Prof. Giuseppe Andrea Buscaino. Since the early beginning of his career, Dario Grossi was fascinated by the study of brain-behavior relationships. To address this topic, between 1975 and 1976, he joined the so-called Milan group, which, at those times, was laying the foundations of neuropsychology in Italy. At his comeback in Naples, Dario Grossi founded a neuropsychology laboratory in the Clinica Neurologica of the University of Naples Federico II, the first in Southern Italy, where many young neurologists were trained (thus establishing a sort of 'Naples' group'). He worked there for more than 20 years intensely, and the lab is still working with renewed personnel. In 2001, he finally moved to the Department of Psychology of the Second University of Naples (now called University of Campania 'Luigi Vanvitelli'), in Caserta, before as an associate professor and then as a full professor. Here he served as the vice-chair and then as the director of the Department until 2018. In Caserta, he promoted a new neuropsychology lab, together with some of his fellows on the 'Naples' group'. He continued his studies in neuropsychology and cognitive neuroscience. His mission of teaching and broadening students' general interest toward neuropsychology materialized, among other enterprises, in the establishment of the School of Specialization in Neuropsychology in 2017, the first in Southern Italy. He served as the director of the school until his retirement. $\mathrm{He}$ was among the founders of the Italian Neuropsychological Society, in which he served as the president from 2013 to 2016. He has been the director of the PhD school on Science for Life and Environment of the University of Campania 'Luigi Vanvitelli' (2010-2012), and the director of the Inter-University Center for Research in Neurosciences (CIRN; 2007-2011). He was a member of the International Neuropsychological Symposium, a worldwide renowned group, since 1982.

Professor Dario Grossi was a gentleman and a humanist in nature. His deep and insightful culture ranged from the ancient Greek philosophers to modern thinkers, from cosmology to epistemology. On these roots, Dario Grossi developed his interests and sensitivity for observing and comprehending human behavior and its relationships with 
the brain. He was a brilliant clinical and behavioral neurologist and a neuropsychologist and a valuable scientist. His attention to patients' signs and symptoms, combined with his jovial disposition and his stunning communicative abilities, made his case descriptions intriguing, surprising, and highly informative, even at a dinner after a national or international meeting. He was able to attract and fascinate a lot of colleagues and students. All of them, all of us who deemed him as an inestimable friend, preserve an indelible memory of Dario Grossi.

\section{Declarations}

Conflict of interest None.

Ethical approval None.

Publisher's note Springer Nature remains neutral with regard to jurisdictional claims in published maps and institutional affiliations. 